International Journal of Pure and Applied Mathematics

Volume 94 No. 2 2014, 251-261

ISSN: 1311-8080 (printed version); ISSN: 1314-3395 (on-line version)

url: http://www.ijpam.eu

doi: http://dx.doi.org/10.12732/ijpam.v94i2.12

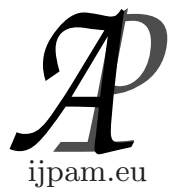

\title{
ON SOME EQUIVALENCE RELATION $\rho$ IN THE CLASS OF BOUNDED SEQUENCES OF POSITIVE REAL NUMBERS
}

\author{
Michał Różański ${ }^{1}$, Damian Słota ${ }^{2} \S$ \\ Marcin Szweda ${ }^{3}$, Roman Wituła ${ }^{4}$ \\ 1,2,3,4 Institute of Mathematics \\ Silesian University of Technology \\ Kaszubska 23, 44-100 Gliwice, POLAND
}

\begin{abstract}
In this paper some equivalence relation in the class of bounded sequences of positive numbers is discussed. This relation enables to understand better the permutations preserving divergence of the same rate, introduced by Johnston. It also gives the possibility to prove, in a new way, the combinatoric characterization of these permutations.
\end{abstract}

AMS Subject Classification: 40A05, 05A05

Key Words: ivergence of series at the same rate, permutation preserving divergence of the same rate

\section{Introduction}

Motivation for preparing this paper was Johnston's paper [3] and the rearrangements that preserve rates of divergence discussed in it. Let us recall, after Johnston, some notation.

Received: May 4, 2014

(C) 2014 Academic Publications, Ltd. url: www.acadpubl.eu

${ }^{\S}$ Correspondence author 
Definition 1. Let $\sum a_{k}$ and $\sum b_{k}$ be the divergent series of positive terms. We say that $\sum a_{k}$ and $\sum b_{k}$ diverge at the same rate if

$$
0<\alpha=\liminf _{n \rightarrow \infty} \frac{\sum_{k=1}^{n} a_{k}}{\sum_{k=1}^{n} b_{k}} \leq \limsup _{n \rightarrow \infty} \frac{\sum_{k=1}^{n} a_{k}}{\sum_{k=1}^{n} b_{k}}=\beta<\infty .
$$

If $\alpha=\beta=1$ in the above relation, then we say that these series are asymptotic and we write $\sum a_{k} \sim \sum b_{k}$.

Definition 2. A permutation $p$ on $\mathbb{N}$ is called the permutation preserving divergence of the same rate if for each divergent series $\sum a_{k}$, with positive and bounded sequence of terms, both series $\sum a_{k}$ and its $p$-rearrangement $\sum a_{p(k)}$ diverge at the same rate.

Johnston observed that the family of all permutations on $\mathbb{N}$ preserving divergence of the same rate forms a group and it is not the normal subgroup of the group of all permutations on $\mathbb{N}$.

Furthermore, he proved the following combinatoric characterization of these permutations.

Theorem 3. Let $p$ be a permutation on $\mathbb{N}$. Then $p$ is a permutation preserving divergence of the same rate if and only if there exists $N=N(p) \in \mathbb{N}$ such that

$$
\operatorname{card}(I \backslash p(I)) \leq N
$$

for every finite interval $I \subset \mathbb{N}$.

In this paper we introduce some equivalence relation $\rho$, defined in the family of bounded sequences of positive numbers, which gives us the possibility to investigate more carefully the permutations preserving divergence of the same rate, presented and discussed by E. Johnston. This relation enables also to obtain easily the Johnston characterization (see Theorem 3 from above) shedding some new light on condition (2) (see Corollary 10 at the end of this paper).

\section{Equivalence Relation $\rho$}

Let $d$ denote the class of bounded sequences of positive real numbers. We introduce in class $d$ relation $\rho$ defined in the following way:

$$
\left\{a_{n}\right\} \rho\left\{b_{n}\right\} \Leftrightarrow \text { for every increasing sequence }\left\{n_{i}\right\} \text { of natural }
$$


numbers both series $\sum a_{n_{i}}$ and $\sum b_{n_{i}}$ are

simultaneously either convergent or divergent,

for every $\left\{a_{n}\right\},\left\{b_{n}\right\} \in d$. One can easily verify that $\rho$ is the equivalence relation in $d$. Let $\left\{a_{n}\right\},\left\{b_{n}\right\} \in d$. If

$$
\left\{\begin{array}{l}
\sum a_{n_{i}}=+\infty \text { and } \sum b_{n_{i}}<+\infty \\
\text { for some increasing sequence }\left\{n_{i}\right\} \subset \mathbb{N},
\end{array}\right.
$$

then sequences $\left\{a_{n}\right\}$ and $\left\{b_{n}\right\}$ belong certainly to different equivalence classes with respect to relation $\rho$ and vice versa. It appears that condition (3) is equivalent with the existence of increasing sequence $\left\{n_{i}\right\} \subset N$ such that

$$
\sum a_{n_{i}}=+\infty \quad \text { and } \quad \lim \frac{a_{n_{i}}}{b_{n_{i}}}=+\infty \quad \text { (see Theorem 4). }
$$

It can be also shown (see Theorem 6 ) that if sequences $\left\{a_{n}\right\},\left\{b_{n}\right\} \in d$ belong to the same equivalence class with respect to relation $\rho$, then there exist the constants $m, M>0, m \leq M$, such that

$$
\sum_{a_{n}>M b_{n}} a_{n}<+\infty \text { and } \sum_{a_{n}<m b_{n}} b_{n}<+\infty,
$$

(if the set of subscripts is empty then we take the sum as equal to 0), from which we get easily that

$$
0<\liminf _{k} \frac{\sum_{n=1}^{k} a_{n}}{\sum_{n=1}^{k} b_{n}} \text { and } \limsup _{k} \frac{\sum_{n=1}^{k} a_{n}}{\sum_{n=1}^{k} b_{n}}<+\infty
$$

(see Theorem 7). We note that the above characterization is not sufficient for belongingness of sequences $\left\{a_{n}\right\}$ and $\left\{b_{n}\right\}$ from $d$ to the same equivalence class with respect to relation $\rho$ (see Remark 9). Whereas if $\left\{a_{n}\right\},\left\{b_{n}\right\} \in d$ and $\liminf \operatorname{in}_{k} \frac{\sum_{n=1}^{k} a_{n}}{\sum_{n=1}^{k} b_{n}}=0$ or $\lim \sup _{k} \frac{\sum_{n=1}^{k} a_{n}}{\sum_{n=1}^{k} b_{n}}=+\infty$, then from (4) it follows that sequences $\left\{a_{n}\right\}$ and $\left\{b_{n}\right\}$ belong to different equivalence classes with respect to relation $\rho$.

We present now the theorems mentioned above. 
Theorem 4. Let $\left\{a_{n}\right\},\left\{b_{n}\right\} \in d$. For existence of an increasing sequence of positive integers $\left\{n_{i}^{\prime}\right\}$, such that $\sum a_{n_{i}^{\prime}}=+\infty$ and $\sum b_{n_{i}^{\prime}}<+\infty$, it is necessary and sufficient that there exists an increasing sequence $\left\{n_{i}\right\} \subset \mathbb{N}$ such that $\sum a_{n_{i}}=+\infty$ and $\lim \frac{a_{n_{i}}}{b_{n_{i}}}=+\infty$.

Proof. Let $c_{n}, d_{n}>0, n \geq 1$. We show that if $\sum c_{n}=+\infty$ and $\sum d_{n}<+\infty$ then there exists an increasing sequence $\left\{n_{i}\right\} \subset \mathbb{N}$ such that

$$
\sum c_{n_{i}}=+\infty \quad \text { and } \quad \lim \frac{c_{n_{i}}}{d_{n_{i}}}=+\infty
$$

First of all, let us notice that then, for each $M>0$, if

$$
\left\{n_{i}\right\}=\left\{n \in \mathbb{N} \mid c_{n} \geq M d_{n}\right\} \text { then } \sum c_{n_{i}}=+\infty .
$$

By this property we define now two auxiliary sets of indices $\left\{t_{n}\right\}$ and $\left\{k_{n}\right\}$. Let

$$
\begin{aligned}
& \mathbb{N}_{1}=\left\{n \in \mathbb{N} \mid c_{n} \geq d_{n}\right\}, \quad k_{1}=\min \mathbb{N}_{1}, \\
& t_{1}=\min \left\{t \in \mathbb{N}_{1} \mid \sum_{k_{1} \leq n \leq t, n \in \mathbb{N}_{1}} c_{n} \geq 1\right\} .
\end{aligned}
$$

In general

$$
\begin{gathered}
\mathbb{N}_{s}=\left\{n \in \mathbb{N} \mid n>t_{s-1} \quad \text { i } \quad c_{n} \geq s d_{n}\right\}, \quad k_{s}=\min \mathbb{N}_{s}, \\
t_{s}=\min \left\{t \in \mathbb{N}_{s} \mid \sum_{k_{s} \leq n \leq t, n \in \mathbb{N}_{s}} c_{n} \geq 1\right\}, \quad \text { for every } s \in \mathbb{N}, s>1 .
\end{gathered}
$$

One can easily verify that the increasing sequence $\left\{n_{i}\right\}$ of all elements of the following set

$$
\left\{n \in \mathbb{N} \mid \text { there exists } s \in \mathbb{N} \text { such that } k_{s} \leq n \leq t_{s} \text { and } n \in \mathbb{N}_{s}\right\}
$$

fulfils conditions (5).

Now, let us assume that there exists an increasing sequence $\left\{n_{i}\right\} \subset \mathbb{N}$ such that $\sum a_{n_{i}}=+\infty$ and $\lim \frac{a_{n_{i}}}{b_{n_{i}}}=+\infty$ and $a_{n_{i}}, b_{n_{i}} \leq 1, i \geq 1$. Let $k_{s}=\min \left\{k \in \mathbb{N} \mid a_{n_{i}} \geq 2^{s} b_{n_{i}}\right.$ for every $\left.i \geq k\right\}, s \geq 1$. We form two auxiliary sequences $\left\{t_{s}\right\}$ and $\left\{l_{s}\right\}$ :

$$
\begin{aligned}
& l_{1}=k_{1}, \\
& t_{1}=\min \left\{t \in \mathbb{N} \mid t \geq l_{1} \text { and } \sum_{i=l_{1}}^{t} a_{n_{i}} \geq 1\right\},
\end{aligned}
$$




$$
\begin{aligned}
& l_{s}=\max \left\{t_{s-1}+1, k_{s}\right\}, \\
& t_{s}=\min \left\{t \in \mathbb{N} \mid t \geq l_{s} \text { and } \sum_{i=l_{s}}^{t} a_{n_{i}} \geq 1\right\},
\end{aligned}
$$

for every $s \in \mathbb{N}, s>1$. Let $\left\{n_{i}^{\prime}\right\}_{i=1}^{\infty}$ be the increasing sequence of all elements of set

$$
\bigcup_{s \in \mathbb{N}}\left\{n_{i} \mid l_{s} \leq i \leq t_{s}\right\}
$$

Then we have

$$
\begin{gathered}
\sum a_{n_{i}^{\prime}}=\sum_{s \geq 1} \sum_{i=l_{s}}^{t_{s}} a_{n_{i}} \geq \sum_{s \geq 1} 1=+\infty \\
\sum b_{n_{i}^{\prime}}=\sum_{s \geq 1} \sum_{i=l_{s}}^{t_{s}} b_{n_{i}} \leq \sum_{s \geq 1} \frac{1}{2^{s}}\left(\sum_{i=l_{s}}^{t_{s}} a_{n_{i}}\right) \leq \sum_{s \geq 1} \frac{2}{2^{s}}=2<+\infty .
\end{gathered}
$$

Corollary 5. Let $a_{n}, b_{n}>0, \lim b_{n}=0$. If $\sum a_{n}=+\infty$, then there exists an increasing sequence $\left\{n_{i}\right\} \subset \mathbb{N}$ such that

$$
\sum a_{n_{i}}=+\infty \text { and } \sum a_{n_{i}} b_{n_{i}}<+\infty .
$$

Proof. It is enough to notice that $\sum a_{n}=+\infty$ and $\lim \frac{a_{n}}{a_{n} b_{n}}=\lim \frac{1}{b_{n}}=+\infty$, and to apply Theorem 4.

Theorem 6. Let $\left\{a_{n}\right\},\left\{b_{n}\right\} \in d, \sum a_{n}=\sum b_{n}=+\infty$. If sequences $\left\{a_{n}\right\}$ and $\left\{b_{n}\right\}$ belong to the same equivalence class with respect to relation $\rho$, then there exists $M>0$ such that $\sum_{a_{n}>M b_{n}} a_{n}<+\infty$ (if $\left\{n \in \mathbb{N} \mid a_{n}>M b_{n}\right\}=\emptyset$, then $\left.\sum_{a_{n}>M b_{n}} a_{n}:=0\right)$.

Proof. Let us suppose that for each $M>0$

$$
\sum_{a_{n}>M b_{n}} a_{n}=+\infty
$$

For simplicity we assume that $a_{n} \leq 1, n \leq 1$. We form two auxiliary sequences $\left\{k_{s}\right\}$ and $\left\{t_{s}\right\}$ of positive integers

$$
k_{1}=\min \left\{k \in \mathbb{N} \mid a_{k}>b_{k}\right\},
$$




$$
\begin{aligned}
& t_{1}=\min \left\{t \in \mathbb{N} \mid t \geq k_{1} \text { and } \sum_{\substack{k_{1} \leq n \leq t \\
a_{n} \geq b_{n}}} a_{n} \geq 1\right\} \text {, } \\
& k_{s}=\min \left\{k \in \mathbb{N} \mid k>t_{s-1} \quad \text { and } \quad a_{k}>2^{s-1} b_{k}\right\} \text {, } \\
& t_{s}=\min \left\{t \in \mathbb{N} \mid t \geq k_{s} \text { and } \sum_{\substack{k_{s} \leq n \leq t \\
a_{n}>2^{s-1} b_{n}}} a_{n} \geq 1\right\} \text {, }
\end{aligned}
$$

for every $s>1$. Let $\left\{n_{i}\right\}$ be the increasing sequence of all elements of set

$$
\bigcup_{s \in \mathbb{N}}\left\{n \in \mathbb{N} \mid k_{s} \leq n \leq t_{s} \quad \text { and } \quad a_{n}>2^{s-1} b_{n}\right\} .
$$

We can easily check that $\sum a_{n_{i}}=+\infty$ and $\sum b_{n_{i}}<+\infty$. Indeed, we have

$$
\begin{gathered}
\sum a_{n_{i}}=\sum_{\substack{s \geq 1 \\
\sum_{s} \leq n \leq t_{s} \\
a_{n}>2^{s-1} b_{n}}} a_{n} \geq \sum_{s \geq 1} 1=+\infty, \\
\sum b_{n_{i}}=\sum_{s \geq 1} \sum_{\substack{k_{s} \leq n \leq t_{s} \\
a_{n}>2^{s-1} b_{n}}} b_{n} \leq \sum_{s \geq 1} \frac{1}{2^{s-1}} \sum_{\substack{k_{s} \leq n \leq t_{s} \\
a_{n}>2^{s-1} b_{n}}} a_{n} \leq \sum_{s \geq 1} \frac{2}{2^{s-1}}=4<+\infty .
\end{gathered}
$$

Thus the sequences $\left\{a_{n}\right\}$ and $\left\{b_{n}\right\}$ belong to the different equivalence classes with respect to relation $\rho$ which contradicts the assumption.

Theorem 7. Let $\left\{a_{n}\right\},\left\{b_{n}\right\} \in d$. If sequences $\left\{a_{n}\right\}$ and $\left\{b_{n}\right\}$ belong to the same equivalence class with respect to relation $\rho$, then

$$
0<\liminf _{k} \frac{\sum_{n=1}^{k} a_{n}}{\sum_{n=1}^{k} b_{n}} \leq \underset{k}{\limsup } \frac{\sum_{n=1}^{k} a_{n}}{\sum_{n=1}^{k} b_{n}}<+\infty
$$

Proof. It is sufficient to confine the considerations to the case $\sum a_{n}=$ $\sum b_{n}=+\infty$. Let $M>0$ be such as in Theorem 6 . Let us put

$$
\sum_{n=1}^{k} a_{n}:= \begin{cases}0 & \text { if }\left\{n \in \mathbb{N} \mid 1 \leq n \leq k \text { and } a_{n}>M b_{n}\right\}=\emptyset \\ \sum_{\substack{1 \leq n \leq k \\ a_{n}>M b_{n}}} a_{n} & \text { otherwise }\end{cases}
$$


and similarly

$$
\sum_{n=1}^{k} * b_{n}:= \begin{cases}0 & \text { if }\left\{n \in \mathbb{N} \mid 1 \leq n \leq k \text { and } a_{n}>M b_{n}\right\}=\emptyset, \\ \sum_{\substack{1 \leq n \leq k \\ a_{n}>M b_{n}}} b_{n} & \text { otherwise. }\end{cases}
$$

Since $\sum_{n \geq 1}{ }^{*} a_{n}<+\infty$, therefore $\sum_{a_{n} \leq M b_{n}} a_{n}=+\infty$ must hold and from this, for sufficiently large $k \in \mathbb{N}$ we get

$$
\frac{\sum_{n=1}^{k} a_{n}}{\sum_{n=1}^{k} b_{n}}=\frac{\sum_{\substack{1 \leq n \leq k \\ a_{n} \leq M b_{n}}} a_{n}+\sum_{n=1}^{k} * a_{n}}{\sum_{\substack{1 \leq n \leq k \\ a_{n} \leq M b_{n}}} b_{n}+\sum_{n=1}^{k} * b_{n}} \leq \frac{\sum_{\substack{1 \leq n \leq k \\ a_{n} \leq M b_{n}}} a_{n}+\sum_{n=1}^{k} * a_{n}}{\sum_{\substack{1 \leq n \leq k \\ a_{n} \leq M b_{n}}} b_{n}} \leq \frac{\sum_{n \geq 1} * a_{n}}{\sum_{\substack{1 \leq n \leq k \\ a_{n} \leq M \\ n}} b_{n}} .
$$

But condition $\sum_{a_{n} \leq M b_{n}} a_{n}=+\infty$ implies that also $\sum_{a_{n} \leq M b_{n}} b_{n}=+\infty$, thus $\lim _{k}\left(\sum_{n=1}^{k} * a_{n}\right) /\left(\sum_{\substack{1 \leq n \leq k \\ a_{n} \leq M b_{n}}} b_{n}\right)=0$, which gives the boundedness of sequence $\left\{\left(\sum_{n=1}^{k} a_{n}\right) /\left(\sum_{n=1}^{k} b_{n}\right)\right\}_{k=1}^{\infty}$. Analogically we prove that sequence

$$
\left\{\left(\sum_{n=1}^{k} b_{n}\right) /\left(\sum_{n=1}^{k} a_{n}\right)\right\}_{k=1}^{\infty}
$$

is bounded as well.

Corollary 8. Let $\left\{a_{n}\right\},\left\{b_{n}\right\} \in d$. If the one of the following conditions hold

$$
\liminf _{k} \frac{\sum_{n=1}^{k} a_{n}}{\sum_{n=1}^{k} b_{n}}=0 \quad \text { or } \quad \limsup _{k} \frac{\sum_{n=1}^{k} a_{n}}{\sum_{n=1}^{k} b_{n}}=+\infty
$$

then the sequences $\left\{a_{n}\right\}$ and $\left\{b_{n}\right\}$ belong to the different equivalence classes with respect to relation $\rho$.

In other words, if $\limsup _{k}\left(\sum_{n=1}^{k} a_{n}\right) /\left(\sum_{n=1}^{k} b_{n}\right)=+\infty$, then there exist the positive integers $\left\{n_{i}\right\}$ such that

$$
\sum a_{n_{i}}=+\infty \text { and } \sum b_{n_{i}}<+\infty .
$$


Remark 9. Theorem 7 cannot be inverted. More precisely, there exist sequences $\left\{a_{n}\right\},\left\{b_{n}\right\} \in d$ satisfying condition (1) and belonging to the different equivalence classes with respect to relation $\rho$. For this purpose let us set

$$
\begin{aligned}
& a_{n}=n^{-1}, \\
& b_{n}= \begin{cases}k^{-1} & \text { if } n=2 k, k \in \mathbb{N}, \\
2^{-n} & \text { if } n \text { is odd }\end{cases}
\end{aligned}
$$

for every $n \in \mathbb{N}$.

We easily check that $\lim _{k}\left(\sum_{n=1}^{k} a_{n}\right) /\left(\sum_{n=1}^{k} b_{n}\right)=1$ and, in spite of this, sequences $\left\{a_{n}\right\}$ and $\left\{b_{n}\right\}$ belong to the different equivalence classes with respect to relation $\rho$. Indeed, we have

$$
\begin{aligned}
& \sum_{k \geq 1} a_{2 k-1}=\sum_{k \geq 1} \frac{1}{2 k-1}=+\infty \\
& \sum_{k \geq 1} b_{2 k-1}=\sum_{k \geq 1} 2^{-2 k+1}<+\infty .
\end{aligned}
$$

\section{Proof of Johnston's Theorem 3}

First we observe that if

$$
\sup \{\operatorname{card}(I \backslash p(I))\}=\infty
$$

where the supremum is taken over all finite intervals $I$ in $\mathbb{N}$, then there exists a sequence $\left\{I_{n}\right\}$ of finite intervals in $\mathbb{N}$ such that

$$
\begin{gathered}
\operatorname{card}\left(I_{n}\right) \rightarrow \infty \text { as } n \rightarrow \infty, \\
I_{n} \cap p\left(I_{n}\right)=\emptyset
\end{gathered}
$$

and $k<l$ for any $k \in I_{n} \cup p\left(I_{n}\right), l \in I_{n+1} \cup p\left(I_{n+1}\right)$ and $n \in \mathbb{N}$.

Hence we get

$$
A \cap p(A)=\emptyset
$$

where $A:=\bigcup_{n \in \mathbb{N}} I_{n}$, and the set $A$ is infinite. This implies that there exists a series $\sum a_{k}$ of positive terms such that $\sum_{k \in A} a_{k}$ is divergent and simultaneously 
the series $\sum_{k \in A} a_{p(k)}$ is convergent, which means that $p$ is not a permutation on $\mathbb{N}$ preserving divergence of the same rate.

On the other hand, if $M \in \mathbb{N}$ and $\operatorname{card}(I \backslash p(I)) \leq M$, for every finite interval $I$ in $\mathbb{N}$, then for every $n \in \mathbb{N}$ there exist the disjoint sets $A_{n}, B_{n} \subset \mathbb{N}$ such that

$$
\begin{gathered}
p([1, n])=\left([1, n] \backslash A_{n}\right) \cup B_{n}, \\
\operatorname{card}\left(A_{n} \cup B_{n}\right)=M .
\end{gathered}
$$

Next, if $\left\{a_{k}\right\}$ is a bounded sequence of positive terms and the series $\sum a_{k}$ is divergent then

$$
\begin{gathered}
\sum_{k=1}^{n} a_{k} \rightarrow \infty \quad \text { as } n \rightarrow \infty, \\
\sum_{k=1}^{n} a_{p(k)}=\sum_{k=1}^{n} a_{k}-\sum_{k \in A_{n}} a_{k}+\sum_{k \in B_{n}} a_{k},
\end{gathered}
$$

which implies that

$$
\left(\sum_{k=1}^{n} a_{p(k)}\right) /\left(\sum_{k=1}^{n} a_{k}\right) \stackrel{n \rightarrow \infty}{\longrightarrow} 1
$$

and it means that $p$ is the permutation preserving divergence of the same rate.

Corollary 10. Let $p$ be a permutation on $\mathbb{N}$. If

$$
\sup \{\operatorname{card}(I \backslash p(I))\}=\infty
$$

where supremum is taken over all finite intervals $I$ in $\mathbb{N}$, then there exists a divergent series $\sum a_{n}$ with $\left\{a_{n}\right\} \in d$ such that the sequences $\left\{a_{n}\right\}$ and $\left\{a_{p(n)}\right\}$ belong to the different equivalence classes with respect to relation $\rho$. We note that condition (7) could be replaced by the following strange condition: there exists an infinite set $A \subset \mathbb{N}$ such that $A \cap p(A)=\emptyset$.

Furthermore, if for every $\left\{a_{n}\right\} \in d$ the sequences $\left\{a_{n}\right\}$ and $\left\{a_{p(n)}\right\}$ belong to the same equivalence class with respect to relation $\rho$, then there exist $N=$ $N(p) \in \mathbb{N}$ such that $p(n)=n$ for every $n \in \mathbb{N}, n \geq N$. 


\section{Final Comments}

The subject matter using or referring to the quotients of the $n$th partial sums of series, discussed here, appears in many papers of various authors (see [1], [2], [4]). With no doubts, this subject represents a very useful technical tool and we think that the results presented in this paper can contribute in consolidating this matter.

Next, from Theorem 3 it follows that every permutation $p$ on $\mathbb{N}$ preserving divergence of the same rate is simultaneously a convergent permutation, i.e. permutation satisfying the following condition: for every convergent real series $\sum a_{n}$ (either complex series or even vector series in complete normed space) the $p$-rearranged series $\sum a_{p(n)}$ is also convergent. It is well know that the convergent permutations preserve also the sum of rearranged series (see [5], [6], [7]). We note that if $q$ is a bijection of $\mathbb{N}$ onto some subset $\mathbb{W}$ of $\mathbb{N}$ with finite complement and the condition (2) holds, then $q$ is a bijection preserving convergence in sense of definition of the convergent permutation $p$ given above. But there is one spectacular difference between these, so called, convergent bijections and convergent permutations. Convergent bijections generally do not preserve the sum of rearranged series.

\section{References}

[1] E. Hensz-Chądzyńska, R. Jajte, A. Paszkiewicz, Random stain, Probab. Math. Statist., 18, No. 1 (1988), 199-218.

[2] D. Holy, L. Matejicka, L. Pinda, Some remarks of faster convergent infinite series, Math. Slovaca, 62, No. 4 (2012), 785-804, doi: 10.2478/s12175-0120045-6.

[3] E.H. Johnston, Rearrangements that preserve rates of divergence, Can. J. Math., 34, No. 4 (1982), 916-920, doi: 10.4153/CJM-1982-064-2.

[4] F. Prus-Wiśniowski, On inclusion between Watermann classes and Chanturyia classes, Tatra Mt. Math. Publ., 19 (2000), 219-227.

[5] R. Wituła, Convergence - preserving functions, Nieuw Arch. Wisk. IV. Ser., 13, No. 1 (1995), 31-35.

[6] R. Wituła, Permutations preserving the sum of rearranged real series, Cent. Eur. J. Math., 11, No. 5 (2013), 956-965, doi: 10.2478/s11533-012-0156-x. 
[7] R. Wituła, Permutations preserving the convergence or the sum of series a survey, in R. Wituła, D. Słota, W. Hołubowski (eds.), Monograph on the Occasion of $100^{\text {th }}$ Birthday Anniversary of Zygmunt Zahorski, Wyd. Pol. Śl., Poland (2014), in press. 
